\title{
Hedgehog-interacting protein is a COPD susceptibility gene: the Rotterdam Study
}

\author{
Y.M.T.A. van Durme*,\#,**, M. Eijgelsheim ${ }^{\#, * *, ~ G . F . ~ J o o s *, ~ A . ~ H o f m a n ~}{ }^{\#, \text {, }}$ \\ A.G. Uitterlinden ${ }^{\#, \uparrow_{,+}}$, G.G. Brusselle ${ }^{*, \#}$ and B.H.Ch. Stricker ${ }^{\#, \uparrow_{,}, \S_{,}, f}$
}

ABSTRACT: The Hedgehog signalling pathway plays an important role in lung morphogenesis and cellular responses to lung injury. A genome-wide association study has demonstrated that two single nucleotide polymorphisms (SNPs) near the Hedgehog-interacting protein (Hip) gene, SNP identifiers rs1828591 and rs13118928, are associated with risk of chronic obstructive pulmonary disease (COPD). The aim of the present study was to validate the observed association between genetic variation near the Hip gene and COPD, and to investigate whether risk estimates were modified by smoking behaviour.

The association between the Hip gene SNPs and COPD was investigated in the Rotterdam Study by logistic regression analyses, adjusted for several covariates. In addition, an association meta-analysis was performed that included data from the genome-wide association study on COPD.

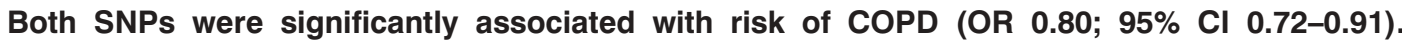
Homozygosity for the minor G allele resulted in a decreased risk of COPD of $\sim 40 \%(95 \% \mathrm{Cl} 0.47-$ 0.78). There was a significant interaction with the number of pack-years of smoking $(p=0.004)$. The meta-analysis yielded an odds ratio for COPD of 0.80 per additional $G$ allele $\left(p=3.4 \times 10^{-9}\right)$.

Genetic variation near the Hip gene was significantly associated with risk of COPD, depending on the number of pack-years of smoking.

KEYWORDS: Chronic obstructive pulmonary disease, epidemiology of chronic obstructive pulmonary disease, genetics, Hedgehog-interacting protein

hronic obstructive pulmonary disease (COPD) is a major worldwide and still increasing health problem [1]. The disease is characterised by progressive airflow limitation, driven by an abnormal inflammatory response of the airways to inhaled particles and fumes [2, 3]. Smoking is the major aetiological factor for COPD in the Western world [1]. However, only $15-25 \%$ of cigarette smokers seem to develop the disease, suggesting the presence of susceptibility genes for COPD [4, 5]. Previous studies have demonstrated familial aggregation of COPD, and some twin studies on pulmonary function have indicated a genetic contribution to clinically relevant parameters, such as forced expiratory volume in $1 \mathrm{~s}$ (FEV1) and forced vital capacity (FVC) [6-8].

The evolutionarily highly conserved Hedgehog (Hh) signalling pathway is crucial to several developmental processes, including lung organogenesis, and is also implicated in the response of the airway epithelium to smoking [9-11]. In cells that respond to $H h$, two proteins are upregulated, i.e. the Hh receptor Patched protein (Ptc) and the vertebrate-specific $H$ h- interacting protein (Hip). Both play an important role in the negative feedback regulation of $H$ h signalling. Loss of Hip function in mouse has already been associated with specific lung defects and results in neonatal lethality [12]. As in mice, dysfunction of the $H h$ pathway during human fetal life is responsible for severe lung malformations [13-15]. In adult life, the $H h$ pathway plays a central role in the repair and regeneration of several tissues, and aberrant activation of this pathway by epithelial stem cells has been linked to various malignancies, including lung cancer $[16,17]$.

Two recent genome-wide association (GWA) studies unexpectedly demonstrated that two single nucleotide polymorphisms (SNPs) near the Hip locus (SNP identifiers rs13118928 and rs1828591) were associated with COPD and lung

\section{AFFILIATIONS}

*Dept of Respiratory Medicine, Ghent University and Ghent University Hospital, Ghent, Belgium. "Depts of Epidemiology, ${ }^{+}$Internal Medicine, ${ }^{f}$ Medical Informatics, Erasmus University Medical Center, "Netherlands Genomics Initiativesponsored Netherlands Consortium for Healthy Ageing, Rotterdam, s Inspectorate of Healthcare, The Hague, The Netherlands.

**Both authors contributed equally to this study.

CORRESPONDENCE

B.H.Ch. Stricker

Dept of Epidemiology

Erasmus Medical Center P0 Box 2040

$3000 \mathrm{CA}$

Rotterdam

The Netherlands

E-mail: b.stricker@erasmusmc.nl

Received:

Aug 142009

Accepted after revision: Nov 172009

First published online:

Dec 082009 
function, respectively, although genome-wide significance levels were not reached for the COPD phenotype [18, 19].

The objective of the current study was to study the abovementioned association between SNPs near the Hip gene and COPD, and to investigate whether smoking or sex modified this association, in a large prospective population-based cohort study with $\sim 20$ yrs of follow-up.

\section{METHODS}

\section{Study population}

The present study forms part of the Rotterdam Study, a population-based cohort study aimed at assessing the occurrence of and risk factors for chronic diseases in the elderly. The objectives and methods of the Rotterdam Study have been described elsewhere [20, 21]. In short, the Rotterdam Study cohort included 7,983 participants aged $\geqslant 55$ yrs living in Ommoord, a well-defined suburb of the city of Rotterdam (the Netherlands). Almost all (99.8\%) of the participants are of Caucasian descent. Baseline data were collected during 19891993. Participants were visited at home at the start of the study for a standardised interview regarding their health status. Since the start of the Rotterdam Study, each participant has visited the research centre every $2-3$ yrs, during which several measurements were performed. In addition, participants were continuously monitored for the onset of major events, which occurred during follow-up through automated linkage with files from general practitioners. Trained research assistants collected information from the medical records of the general practitioners, hospitals and nursing homes. The medical ethics committee of the Erasmus Medical Center (Rotterdam) approved the study. Participants gave written informed consent and permission to retrieve information from treating physicians.

\section{Spirometry}

Spirometry was performed by trained paramedical personnel using a SpiroPro® portable spirometer (Erich Jaeger, Hoechberg, Germany) according to American Thoracic Society (ATS)/ European Respiratory Society (ERS) guidelines [22]. FEV1, FVC and FEV1/FVC ratio were measured; the spirogram (volume-time curve) and maximal expiratory flow-volume curve were also recorded. Spirometry procedures that yielded results that did not meet ATS/ERS criteria for acceptability and reproducibility were classified as not interpretable [23].

\section{Patient identification and validation}

For the validation of the COPD cases, access was available to hospital discharge letters, files from general practitioners, spirometry reports and pharmacy dispensing data for patients participating in the Rotterdam Study, as previously described [23]. Spirometry was performed in 3,550 participants in the context of the Rotterdam cohort study. In addition, throughout the entire study period, spirometry was also performed on clinical indication by respiratory specialists and specialists in internal medicine with a subspeciality in respiratory medicine. In the absence of spirometry, all medical information on subjects who used respiratory medication for $\geqslant 6$ months (anatomical therapeutic chemical classification system codes R03AC, R03AK, R03BA, R03BB, R03CC and R03DA) [24] and all hospital discharge letters or mortality reports with a coded diagnosis of COPD (J40-J44 (International Statistical
Classification of Diseases and Related Health Problems, 10th revision)) [25] were reviewed. Drug use was only used for potential case finding and not for making the diagnosis of COPD. In the case of confirmed COPD, the day of first drug use was used as an indicator of the date of onset of COPD.

The diagnosis of COPD was classified as definite or probable. Definite COPD was defined by moderate-to-severe obstructive spirometry results (FEV1/FVC of $<0.7$ and FEV1 of $<80 \%$ of the predicted value) and/or as COPD diagnosed by a specialist in internal medicine (mainly respiratory physicians or those with a subspecialty in respiratory medicine) based upon the combined results of clinical history, physical examination and spirometry. Probable COPD was defined by mild obstructive spirometry results (FEV1/FVC of $<0.7$ and FEV1 of $\geqslant 80 \%$ pred) and/or as COPD diagnosed by a physician in another medical speciality (e.g. a general practitioner).

The index date was defined as the date of diagnosis of COPD found in the medical reports, the date of a first prescription of COPD medication or the date of the obstructive lung function examination, whichever came first.

\section{Genotyping}

The Illumina Infinium II HumanHap550K BeadChip (version 3; Illumina, San Diego, CA, USA) was used for genotyping 6,449 participants with available DNA in the Rotterdam Study, as previously described [26]. Genotyping was performed at the Dept of Internal Medicine of Erasmus Medical Center using BeadStudio software (version 0.3.10.14; Illumina) for genotyping calling. Participants with a call rate of $<97.5 \%$, excess autosomal heterozygosity, sex mismatch or outlying identityby-state clustering estimates were excluded. A total of 5,974 participants were successfully genotyped and passed quality control. Genotype information on two specific SNPs near the Hip gene located on chromosome 4 (rs13118928 and rs1828591) was extracted from these data.

\section{Statistical analyses}

The demographic and clinical characteristics of the study population are expressed as median with interquartile range (IQR) in the text.

First, the association of rs13118928 and rs1828591 with COPD was studied within the Rotterdam Study. All COPD cases, prevalent and incident, were used for analyses in order to obtain maximal power. Consequently, logistic regression analyses were performed because Cox's proportional hazards analysis was not possible with prevalent cases at baseline. Both SNPs were tested for Hardy-Weinberg equilibrium using Chi-squared statistics. A power calculation using Quanto (University of Southern California, Los Angeles, CA, USA), based on the combined odds ratio (OR) of 0.7 of the Bergen case-control cohort and the National Emphysema Treatment Trial (NETT)/ Normative Aging Study (NAS) population (combined $\beta=-0.332$ ) from the study of PILLAI et al. [18], showed that it was possible to demonstrate an OR for COPD of 0.7 with $>99 \%$ power at a two-sided $\alpha$ of 0.05 [27]. Additive and genotype-based logistic regression models, adjusted for age at baseline, sex, smoking behaviour (ever- and neversmoker) and the total number of pack-years of smoking, were used to calculate ORs and 95\% confidence intervals (CIs). 
In the additive genetic model, the categorical variable of the number of variant alleles (homozygous wild type (0 variant alleles); heterozygous (1 variant allele); homozygous variant allele ( 2 variant alleles)) was included as a continuous value in the logistic regression model in order to calculate trends.

The following covariates were considered as potential confounders or effect modifiers: age, sex, smoking behaviour, and the total number of pack-years of smoking. Smoking status was self-reported and categorised as ever- or never-smoker. The category of ever-smoker included both current and former smokers. The number of pack-years was computed from the duration of smoking (in years) multiplied by the number of cigarettes smoked daily divided by 20 . The number of packyears of smoking of each study participant was calculated using prospectively collected data regarding the smoking behaviour of the cohort members, collected during the first, second and third follow-up visits of the Rotterdam Study.

Participants with asthma or a spirometry result suggestive of restrictive lung disease were excluded from the analyses. Interaction terms and subanalyses were introduced to explore potential effect modification by sex and tertiles of pack-years of smoking. A p-value below the conventional level of significance $(\mathrm{p}<0.05)$ was considered significant.

Secondly, an inverse-variance-weighted meta-analysis was performed. This analysis combined the results obtained to the recently published data of PILLAI et al. [18]. The regression coefficients and SEM were calculated on the basis of the reported ORs and p-values from the Bergen case-control cohort and NETT/NAS data from the recent GWA study of PILlai et al. [18].

The results of the association meta-analysis were calculated using Excel version 2007 (Microsoft Office). All other statistical analyses were performed using SPSS for Windows version 15 (SPSS, Inc., Chicago, IL, USA).

\section{RESULTS}

\section{Baseline characteristics of the study population}

Within the Rotterdam Study source population of 7,983 subjects, there were 928 patients with validated prevalent or incident COPD [23]. Both SNPs, rs13118928 and rs1828591, were successfully genotyped in 5,974 out of the 7,983 participants for whom DNA was available, including 742 COPD cases. A total of 256 study participants with asthma or a spirometry result suggestive of restrictive pulmonary disease were excluded (fig. 1). Overall, the median age of the study population at the start of the study was 68.4 yrs (IQR 14 yrs), and $59 \%$ were female. Additional clinical characteristics are shown in table 1 .

\section{Association between variation near the Hip gene and COPD in the Rotterdam Study}

Both SNPs studied, rs13118928 and rs1828591, were in HardyWeinberg equilibrium (rs13118928: $\chi^{2}=1.413 ; \quad p=0.235$; rs1828591: $\left.\chi^{2}=1.215 ; \mathrm{p}=0.270\right)$ and in perfect linkage disequilibrium $\left(r^{2}=1.00\right.$ in HapMap CEU reference samples (Utah residents of Northern and Western European descent)). Owing to the high linkage between the two SNPs, analyses yielded the same results and thus, for reasons of simplicity, only the results for the rs13118928 SNP are shown in the text and tables.
Under an additive genetic model, there was a significant association between both SNPs and the risk of COPD with an OR of 0.80 per additional guanine (G) allele (95\% CI 0.72-0.91; $\mathrm{p}<0.001)$. Genotypic analysis showed that homozygosity for the minor $G$ allele was associated with a significantly decreased risk of COPD of $\sim 40 \%$ (OR 0.60 ; 95\% CI $0.47-0.78$; $\mathrm{p}<0.001$ ) (table 2). The decrease in risk remained significant when the analyses were restricted to those participants with an available spirometric examination during follow-up in the Rotterdam Study (OR 0.60; 95\% CI 0.43-0.89; $\mathrm{p}=0.011$ ) (data not shown).

There was no interaction with sex $(p=0.872)$, but a significant interaction effect was present with the total number of packyears of smoking $(p=0.004)$. Additional subanalyses demonstrated that the protective effect of having one or more variant alleles was most pronounced for smoking participants in the highest tertile of pack-years of smoking ( $>36$ pack-yrs), with an OR of $0.80(95 \%$ CI $0.62-0.93 ; p=0.007)$ versus an OR of 0.90 $(95 \%$ CI $0.67-1.09 ; \mathrm{p}=0.209)$ in those who had never smoked (table 3).

\section{Association between variation near the Hip gene and COPD: meta-analysis with published results}

The original $\mathrm{p}$-value, including the Bergen case-control cohort, the International COPD Genetics Network (ICGN) population and the NETT/NAS data, was $1.7 \times 10^{-7}$. Since it was not possible to obtain $\beta$ estimates and SEM for the ICGN population, the $\mathrm{p}$-value for the combination of the Bergen and NETT/NAS data was also calculated. This resulted in a p-value of $1.0 \times 10^{-6}$. The combination of the Rotterdam Study results with the Bergen and NETT/NAS results yielded an overall OR of 0.80 per additional G allele (95\% CI $0.70-0.84$ ), with a p-value of $3.4 \times 10^{-9}$, which is significant at a genomewide level (table 4; fig. 2).

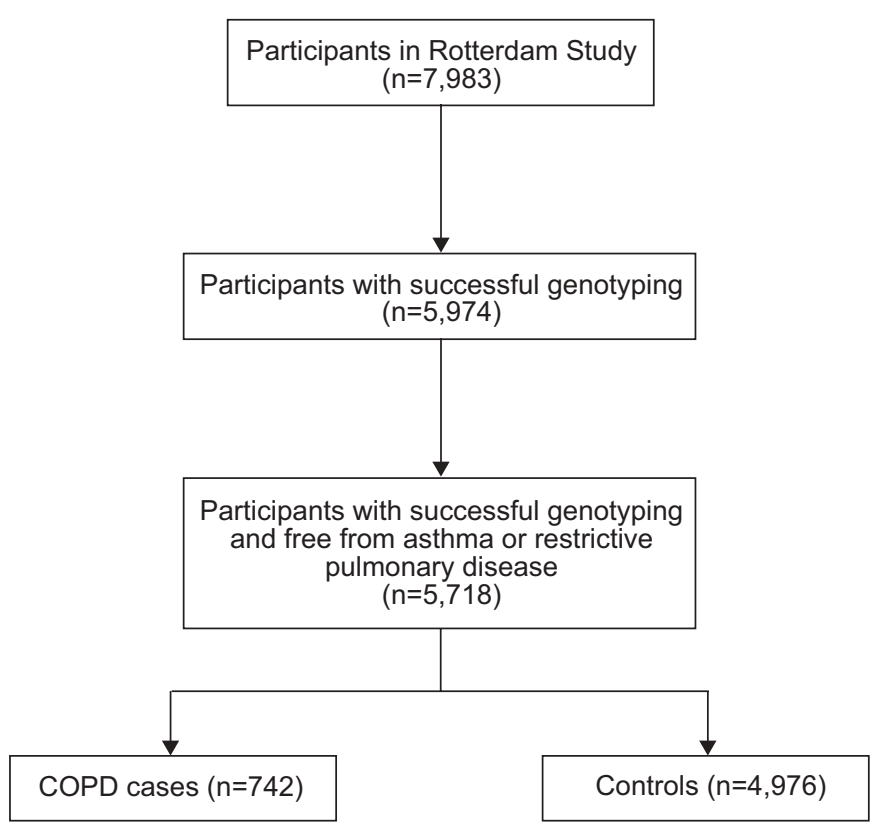

FIGURE 1. Study design. COPD: chronic obstructive pulmonary disease 


\begin{tabular}{|c|c|c|c|c|}
\hline \multirow[t]{2}{*}{ TABLE 1} & \multicolumn{4}{|c|}{$\begin{array}{l}\text { Baseline characteristics of the Rotterdam Study } \\
\text { population }\end{array}$} \\
\hline & & Total & Cases & Controls \\
\hline \multicolumn{2}{|l|}{ Subjects $n$} & 5718 & 742 & 4976 \\
\hline \multicolumn{2}{|l|}{ Age yrs } & $68.4(14)$ & $67.0(10)$ & $68.8(14)$ \\
\hline \multicolumn{2}{|c|}{ Females } & 3397 (59) & $315(43)$ & $3082(62)$ \\
\hline \multicolumn{5}{|c|}{ Smoking status } \\
\hline \multicolumn{2}{|c|}{ Current smoker } & $1290(23)$ & $300(40)$ & $990(20)$ \\
\hline \multicolumn{2}{|c|}{ Former smoker } & $2311(40)$ & $325(44)$ & $1986(40)$ \\
\hline \multicolumn{2}{|c|}{ Never-smoker } & $1960(34)$ & $111(15)$ & $1849(37)$ \\
\hline \multicolumn{2}{|c|}{ Missing data } & $157(3)$ & $6(1)$ & 151 (3) \\
\hline \multicolumn{2}{|c|}{$\begin{array}{l}\text { Smoking history pack-yrs } \\
\text { rs13118928 }\end{array}$} & $9.0(30)$ & $27.8(32)$ & $6.8(28)$ \\
\hline \multicolumn{2}{|c|}{ AA } & 2099 (37) & $294(40)$ & $1805(36)$ \\
\hline \multicolumn{2}{|l|}{$A G$} & $2698(47)$ & $359(49)$ & $2339(47)$ \\
\hline \multicolumn{2}{|l|}{$\mathrm{GG}$} & $921(16)$ & $89(12)$ & $832(17)$ \\
\hline \multicolumn{5}{|l|}{ rs1828591 } \\
\hline \multicolumn{2}{|l|}{ AA } & 2092 (37) & $293(40)$ & 1799 (36) \\
\hline \multicolumn{2}{|l|}{$A G$} & $2703(47)$ & $360(49)$ & $2343(47)$ \\
\hline \multicolumn{2}{|l|}{$\mathrm{GG}$} & $923(16)$ & $89(12)$ & $834(17)$ \\
\hline
\end{tabular}

Data are presented as $\mathrm{n}(\%)$, except for age and smoking history pack-yrs which are presented as median (interquartile range), unless otherwise indicated. A: adenine; G: guanine.

\section{DISCUSSION}

The results of the present population-based prospective cohort study confirm the recently observed association between genetic variation around the Hip gene and COPD, as described by PILLAI et al. [18]. It was found that the minor G allele of the rs13118928 and rs1828591 SNPs is associated with a significantly decreased risk of COPD. The risk seemed to be dependent upon the smoking behaviour of the participants, since the decrease in risk was strongest in subjects who had smoked for $>30$ pack-yrs at the start of the Rotterdam Study.

The $H h$ signalling pathway is an evolutionarily highly conserved signalling cascade that is crucial to several developmental processes [10,11]. In mammals, there are three $\mathrm{Hh}$ proteins: Sonic, Indian, and Desert. Sonic $H h$ specifically targets the lung and is expressed in the lung epithelium during embryogenesis [28]. Signalling is initiated by binding of one of the Hh proteins to the 12-pass transmembrane protein receptor Ptc, which is required to inhibit the activity of Smoothened (Smo), a seven-pass transmembrane protein, which is responsible for regulation of the zinc-finger-containing glioma-associated oncogene homologue (Gli) transcriptional effectors, Gli1, Gli2 and Gli3 [13, 29]. Upon binding of $H h$ to Ptc, the Ptc-mediated inhibition of Smo is relieved [30, 31]. This triggers a cascade that finally results in activation of the Gli family and transcription of $H h$ target genes, including Ptc and Gli itself [32]. The availability of the $H h$ ligands is regulated by expression of Hip. The Hip protein was initially discovered by screening murine cDNA expression for proteins that bound to Sonic Hh. The Hip gene, located on chromosome 4 (4q31.21-4q31.3), encodes a transmembrane glycoprotein that binds all three $H h$ proteins with an affinity equal to that of Ptc and is upregulated upon $\mathrm{Hh}$ signalling [28]. Hip is expressed in

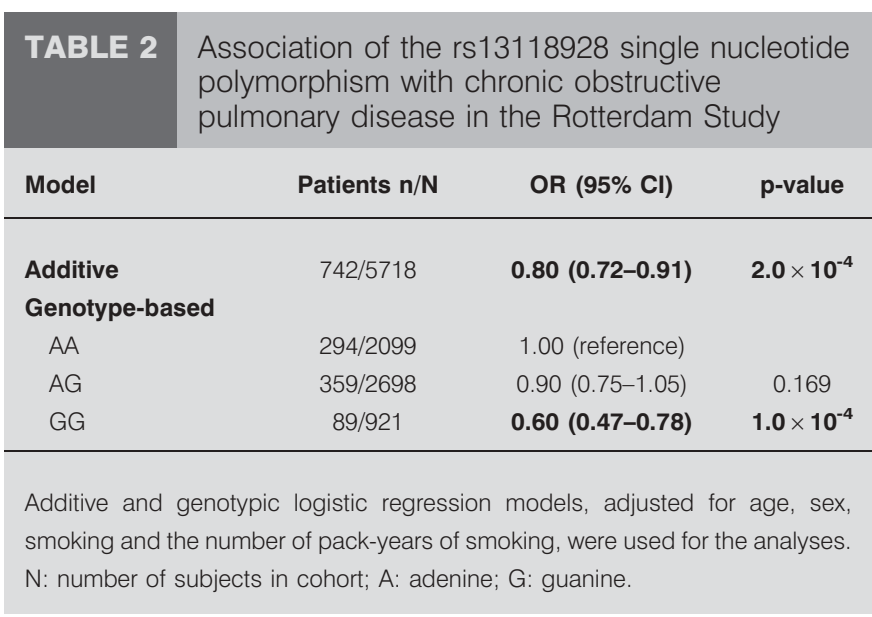

all $H h$ target tissues and acts by internalising and degrading the Hh protein. Similar to Ptc, Hip thus serves as a natural antagonist of the $H h$ pathway.

In this way, alterations in the Hip gene can result in altered gene expression of a functional protein, or in a nonfunctional protein and upregulation of the Hh signalling pathway.

Loss of Hip function in mice during fetal life has been associated with specific lung defects resulting in respiratory failure and neonatal lethality [12]. In adult animals, downregulation of Hip has been reported in murine lung cancer cell lines [33]. As in rodents, dysfunction of the $H h$ pathway during fetal life in humans is responsible for severe lung malformations [13-15]. In adult life, the $H h$ pathway plays a central role in the repair and regeneration of several human tissues, and aberrant activation of this pathway by epithelial stem cells has been linked to various malignancies, including lung cancer $[16,17]$. In addition, Hip seemed to be downregulated in human nonsmall cell lung cancer tissues and endothelial cells during angiogenesis [33].

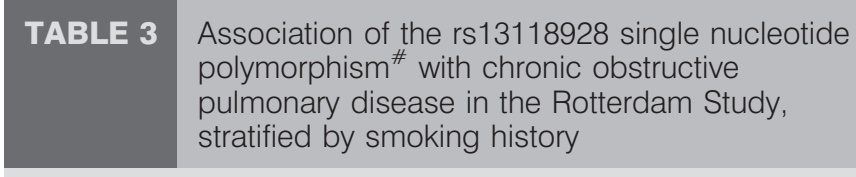

\begin{tabular}{lccc} 
Smoking history pack-yrs & Patients $\mathbf{n} / \mathbf{N}$ & OR $(\mathbf{9 5} \% \mathbf{C l})$ & p-value \\
\hline $\mathbf{0}$ & $111 / 1960$ & $0.90(0.67-1.09)$ & 0.209 \\
$>\mathbf{0}-\leqslant \mathbf{1 5}$ & $114 / 1129$ & $0.80(0.61-1.08)$ & 0.154 \\
$>\mathbf{1 5}-\leqslant \mathbf{3 6}$ & $203 / 1082$ & $0.90(0.70-1.09)$ & 0.224 \\
$>\mathbf{3 6}$ & $277 / 1095$ & $\mathbf{0 . 8 0}(\mathbf{0 . 6 2}-\mathbf{0 . 9 3})$ & $\mathbf{0 . 0 0 7}$ \\
\hline
\end{tabular}

Additive logistic regression models, adjusted for age and sex, were used for the analyses. The number of pack-years of smoking was calculated based on information regarding the smoking behaviour of each study participant, collected prospectively at each interview during the three Rotterdam Study follow-up visits. $\mathrm{N}$ : number of subjects in cohort. ${ }^{\#}$ : near the Hedgehoginteracting protein gene; ${ }^{\prime}$ : categories based on the $33^{\text {rd }}$ percentile of the data in smokers. 


\begin{tabular}{|c|c|c|c|}
\hline TABLE 4 & \multicolumn{3}{|c|}{ 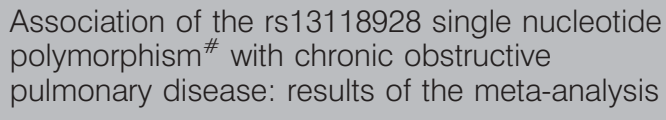 } \\
\hline Sample & & OR (95\% Cl) & $\mathrm{p}$-value \\
\hline \multicolumn{2}{|c|}{ Rotterdam Study } & $0.81(0.72-0.91)$ & $<0.001$ \\
\hline \multicolumn{2}{|c|}{ Bergen cohort } & $0.73(0.62-0.86)$ & $<0.001$ \\
\hline \multicolumn{2}{|c|}{ NETT/NAS } & $0.71(0.56-0.88)$ & 0.002 \\
\hline \multicolumn{2}{|l|}{ Overall } & $0.77(0.70-0.84)$ & $3.43 \times 10^{-9}$ \\
\hline \multicolumn{4}{|c|}{ 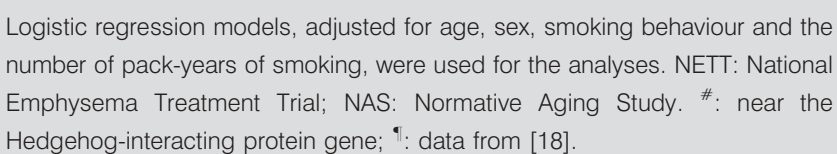 } \\
\hline
\end{tabular}

The results of the present study and the two previous GWA reports demonstrate that variants near the Hip gene influence the risk of COPD. Although it is not clear which regulatory effect explains this association, several hypotheses are possible. First, MADISON et al. [34] demonstrated that ectopic expression of Hip in mice during fetal life was associated with expanded smooth muscle in the intestinal epithelium [31]. If the same effect were present in the small airways, then it could be speculated that changes in Hip influence the thickness of the airway muscle layer and induce more or less airflow limitation. Secondly, a study of POGACH et al. [35] demonstrated that several developmental pathways in the lungs of adult mice, including $H h$, reactivate for tissue repair following hyperoxia. In addition, others have shown that embryogenic signalling pathways, such as $H$, are activated in human bronchial epithelial cells exposed to cigarette smoke [36].

The prevalence, morbidity and mortality of COPD in females are increasing [37]. In addition, some reports suggest that females may be at greater risk of developing severe early-onset COPD due to sex differences in the metabolism of cigarette smoke [38]. However, the results of these studies remain controversial since differences in lung development, smoking behaviour, diagnosis and treatment of COPD are possible confounders. Since sex can be of interest in the pathogenesis of COPD, a potential interaction effect of sex on the observed association between variation near the Hip gene and COPD was tested for. However, no significant influence was found $(\mathrm{p}=0.872)$.

The results of these two studies raise the possibility that the $\mathrm{Hh}$ pathway is involved in the response of the airway epithelium to tissue injury, and that alterations in Hh signalling can lead to susceptibility to COPD, depending upon the severity of the smoking behaviour. Thirdly, since a recent GWA study investigating genetic variants that influence adult height identified several relevant genes in the Hh signalling pathway (Indian Hh, Hip and Ptc), height could be considered a potential confounder for the observed association between Hip and COPD [39]. However, additional analyses including height as a covariate did not influence the present estimates (data not shown).

The strengths of the present study are the high-quality information available regarding exposures prior to outcome,

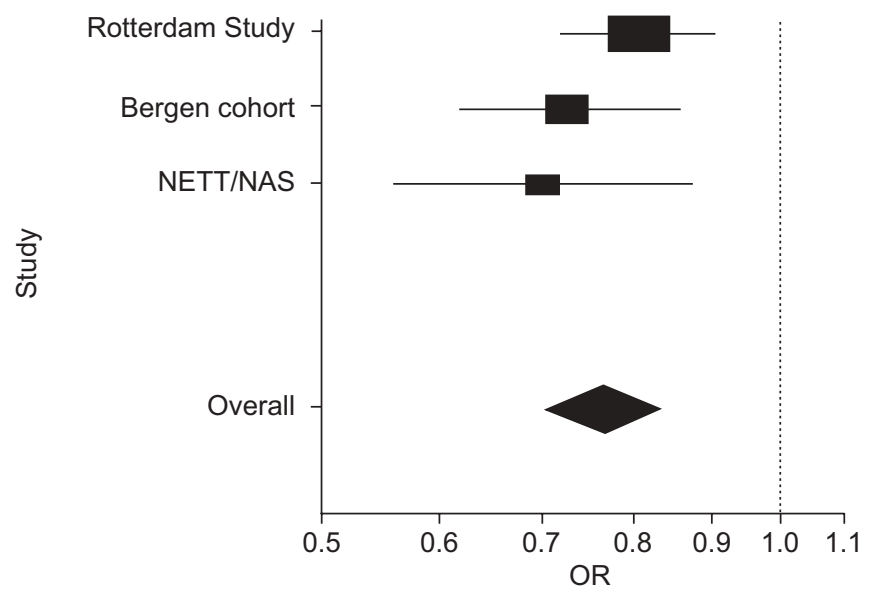

FIGURE 2. Forest plot demonstrating the odds ratios (ORs) for the association between the rs13118928 single nucleotide polymorphism near the Hedgehoginteracting protein gene with chronic obstructive pulmonary disease in the metaanalysis. Data are presented as adjusted ORs ( $\mathbf{\square}$ (size reflects weighting)), using a fixed-effects model, and 95\% confidence interval (horizontal bars). The centre of the diamond indicates the combined mean effect of the studies (OR 0.77) and its extremities the $95 \%$ confidence interval $(0.70-0.84)(\cdots \cdots$ : line of no effect). NETT: National Emphysema Treatment Trial; NAS: Normative Aging Study.

with prospective data collection, the general population-based setting, the large number of subjects that participated in the Rotterdam Study and the long duration of follow-up. The high response rate and virtually complete follow-up for each participant makes information and selection bias unlikely for these data. A power analysis showed that the present study was of sufficient size to distinguish between the proposed effect and no effect. The study was conducted in a data set independent from the original GWA study.

A similar significant protective risk was demonstrated with the same SNPs, and the combined analysis led to a smaller p-value than in the original report, below the stringent threshold frequently used to define genome-wide significance. Hence the present study satisfies all criteria suggested by the National Cancer Institute/National Human Genome Research Institute Working Group on Replication in Association Studies for establishing positive replication [40].

As for all other association studies, no conclusions regarding causality or the mechanism through which the associated SNPs influence COPD risk can be formed. It is possible that the two SNPs studied are not the causal variants, but are in linkage disequilibrium with other polymorphisms that are responsible for the increased COPD risk. Since the SNPs are not situated within the Hip gene and do not seem to exhibit a strong linkage with other SNPs in the Hip gene itself, a regulatory function is possible. Additional functional and translational research examining the role of Hip in COPD is certainly warranted.

In conclusion, the present study confirms the previously reported association between two SNPs near the Hip gene and COPD in a Caucasian population-based cohort study. In addition, a potential gene-by-environment interaction was identified, since the association was most apparent in heavily smoking participants. 


\section{SUPPORT STATEMENT}

The Rotterdam Study is supported by the Erasmus University Medical Center (Rotterdam), Erasmus University (Rotterdam), Netherlands Organization for Scientific Research (NWO; The Hague), Netherlands Organization for Health Research and Development (The Hague), Research Institute for Diseases in the Elderly (The Hague), Ministry of Education, Culture and Science (The Hague) and Ministry of Health, Welfare and Sport (The Hague) (all the Netherlands). This study ?was supported by NWO grants 904-61-093 and 918-46-615, and Netherlands Genomics Initiative/NWO project number 050-060-810. Y.M.T.A van Durme received a travel grant from the Belgian Thoracic Society and is a doctoral research fellow of the Fund for Scientific Research - Flanders (Brussels, Belgium). M. Eijgelsheim is funded by Netherlands Heart Foundation (The Hague, the Netherlands) project number 2007B221.

\section{STATEMENT OF INTEREST}

A statement of interest for G.G. Brusselle can be found at www.erj. ersjournals.com $/ \mathrm{misc} /$ statements.dtl

\section{ACKNOWLEDGEMENTS}

The authors thank J. Verkroost (Erasmus University Medical Center, Rotterdam, the Netherlands) for outstanding work in preparing the data set for the validation of the COPD cases, and G. Van Pottelberge (Ghent University, Ghent, Belgium) for helping to validate the COPD cases. We also thank J. Heeringa (Erasmus University Medical Center), the research assistants and all our other colleagues in the Ommoord research centre (Erasmus University Medical Center) for their efforts in the data collection.

\section{REFERENCES}

1 Mannino DM, Buist AS. Global burden of COPD: risk factors, prevalence, and future trends. Lancet 2007; 370: 765-773.

2 Pauwels RA, Rabe KF. Burden and clinical features of chronic obstructive pulmonary disease (COPD). Lancet 2004; 364: 613-620.

3 Rabe KF, Beghe B, Luppi F, et al. Update in chronic obstructive pulmonary disease 2006. Am J Respir Crit Care Med 2007; 175: 1222-1232.

4 Lokke A, Lange P, Scharling H, et al. Developing COPD: a 25 year follow up study of the general population. Thorax 2006; 61: 935-939.

5 Silverman EK. Progress in chronic obstructive pulmonary disease genetics. Proc Am Thorac Soc 2006; 3: 405-408.

6 McCloskey SC, Patel BD, Hinchliffe SJ, et al. Siblings of patients with severe chronic obstructive pulmonary disease have a significant risk of airflow obstruction. Am J Respir Crit Care Med 2001; 164: 1419-1424.

7 Silverman EK, Chapman HA, Drazen JM, et al. Genetic epidemiology of severe, early-onset chronic obstructive pulmonary disease. Risk to relatives for airflow obstruction and chronic bronchitis. Am J Respir Crit Care Med 1998; 157: 1770-1778.

8 Chen Y. Genetics and pulmonary medicine.10: Genetic epidemiology of pulmonary function. Thorax 1999; 54: 818-824.

9 Chen MH, Wilson CW, Chuang PT. SnapShot: hedgehog signaling pathway. Cell 2007; 130: 386.

10 van Tuyl M, Post M. From fruitflies to mammals: mechanisms of signalling via the Sonic hedgehog pathway in lung development. Respir Res 2000; 1: 30-35.

11 Warburton D, Bellusci S, De Langhe $\mathrm{S}$, et al. Molecular mechanisms of early lung specification and branching morphogenesis. Pediatr Res 2005; 57: 26R-37R.
12 Chuang PT, Kawcak T, McMahon AP. Feedback control of mammalian Hedgehog signaling by the Hedgehog-binding protein, Hip1, modulates Fgf signaling during branching morphogenesis of the lung. Genes Dev 2003; 17: 342-347.

13 Oldak M, Grzela T, Lazarczyk M, et al. Clinical aspects of disrupted Hedgehog signaling. Int J Mol Med 2001; 8: 445-452.

14 Villavicencio EH, Walterhouse DO, Iannaccone PM. The Sonic hedgehog-Patched-Gli pathway in human development and disease. Am J Hum Genet 2000; 67: 1047-1054.

15 Whitsett JA, Wert SE, Trapnell BC. Genetic disorders influencing lung formation and function at birth. Hum Mol Genet 2004; 13: R207-R215.

16 Rubin LL, de Sauvage FJ. Targeting the Hedgehog pathway in cancer. Nat Rev Drug Discov 2006; 5: 1026-1033.

17 Watkins DN, Berman DM, Burkholder SG, et al. Hedgehog signalling within airway epithelial progenitors and in small-cell lung cancer. Nature 2003; 422: 313-317.

18 Pillai SG, Ge D, Zhu G, et al. A genome-wide association study in chronic obstructive pulmonary disease (COPD): identification of two major susceptibility loci. PLoS Genet 2009; 5: e1000421.

19 Wilk JB, Chen TH, Gottlieb DJ, et al. A genome-wide association study of pulmonary function measures in the Framingham Heart Study. PLoS Genet 2009; 5: e1000429.

20 Hofman A, Grobbee DE, Dejong PTVM, et al. Determinants of disease and disability in the elderly - the Rotterdam Elderly Study. Eur J Epidemiol 1991; 7: 403-422.

21 Hofman A, Breteler MM, van Duijn CM, et al. The Rotterdam Study: 2010 objectives and design update. Eur J Epidemiol 2009; 24: 553-572.

22 Celli BR, MacNee W. Standards for the diagnosis and treatment of patients with COPD: a summary of the ATS/ERS position paper. Eur Respir J 2004; 23: 932-946.

23 van Durme YM, Verhamme KM, Stijnen T, et al. Prevalence, incidence, and lifetime risk for the development of COPD in the elderly: the Rotterdam Study. Chest 2009; 135: 368-377.

24 World Health Organization, Anatomical Therapeutic Chemical Classification System. Geneva, World Health Organization, 1996.

25 World Health Organization, International Statistical Classification of Diseases and Related Health Problems, 10th revision. Geneva, World Health Organization, 1992.

26 Newton-Cheh C, Eijgelsheim M, Rice KM, et al. Common variants at ten loci influence QT interval duration in the QTGEN Study. Nat Genet 2009; 41: 399-406.

27 Purcell S, Cherny SS, Sham PC. Genetic Power Calculator: design of linkage and association genetic mapping studies of complex traits. Bioinformatics 2003; 19: 149-150.

28 Chuang PT, McMahon AP. Vertebrate Hedgehog signalling modulated by induction of a Hedgehog-binding protein. Nature 1999; 397: 617-621.

29 McMahon AP. More surprises in the Hedgehog signaling pathway. Cell 2000; 100: 185-188.

30 Ingham PW. Hedgehog signalling. Curr Biol 2008; 18: R238-R241.

31 Parkin CA, Ingham PW. The adventures of Sonic hedgehog in development and repair. I. Hedgehog signaling in gastrointestinal development and disease. Am J Physiol Gastrointest Liver Physiol 2008; 294: G363-G367.

32 Bak M, Hansen C, Friis HK, et al. The human hedgehog-interacting protein gene: structure and chromosome mapping to $4 \mathrm{q} 31.21 \rightarrow \mathrm{q} 31.3$. Cytogenet Cell Genet 2001; 92: 300-303.

33 Olsen CL, Hsu PP, Glienke J, et al. Hedgehog-interacting protein is highly expressed in endothelial cells but down-regulated during angiogenesis and in several human tumors. BMC Cancer 2004; 4: 43.

34 Madison BB, Braunstein K, Kuizon E, et al. Epithelial hedgehog signals pattern the intestinal crypt-villus axis. Development 2005; 132: 279-289. 
35 Pogach MS, Cao Y, Millien G, et al. Key developmental regulators change during hyperoxia-induced injury and recovery in adult mouse lung. J Cell Biochem 2007; 100: 1415-1429.

36 Lemjabbar-Alaoui H, Dasari V, Sidhu SS, et al. Wnt and Hedgehog are critical mediators of cigarette smoke-induced lung cancer. PLoS One 2006; 1: e93.

37 Han MK, Postma D, Mannino DM, et al. Gender and chronic obstructive pulmonary disease: why it matters. Am J Respir Crit Care Med 2007; 176: 1179-1184.
38 Prescott E, Bjerg AM, Andersen PK, et al. Gender difference in smoking effects on lung function and risk of hospitalization for COPD: results from a Danish longitudinal population study. Eur Respir J 1997; 10: 822-827.

39 Weedon $\mathrm{MN}$, Lango $\mathrm{H}$, Lindgren $\mathrm{CM}$, et al. Genome-wide association analysis identifies 20 loci that influence adult height Nat Genet 2008; 40: 575-583.

40 Chanock SJ, Manolio T, Boehnke M, et al. Replicating genotypephenotype associations. Nature 2007; 447: 655-660. 